\title{
Knowledge Among Nursing Students on Zika Preparedness
}

\author{
Vasundharaa S Nair ${ }^{1 *}$, Kasi Sekar ${ }^{2}$ and Priya Treesa Thomas ${ }^{3}$ \\ ${ }^{1}$ PhD Scholar, Department of Psychiatric Social Work, NIMHANS \\ ${ }^{2}$ Professor, Department of Psychiatric Social work, NIMHANS \\ ${ }^{3}$ Associate Professor, Department of Psychiatric Social Work, NIMHANS \\ *Corresponding Author: Vasundharaa S Nair, PhD Scholar, Department of \\ Psychiatric social work, NIMHANS.
}

Received: February 24, 2020

Published: February 28, 2020

(C) All rights are reserved by Vasundharaa $\mathbf{S}$

Nair., et al.

\section{Abstract}

Objective: ZIKA a biological hazard with severe burden affects the well-being and quality of life of vulnerable populations both at micro and macro level. The present research is aimed at preparedness among health care professionals towards this impending challenge.

Methods: National Institute of Mental Health and Neurosciences, a premier institute for neuro-psychiatric disorders formed the universe for the study. Total sample survey of 285 Undergraduate Nursing students using the WHO-KAP schedule was administered after obtaining informed consent. Their responses were content analysed.

Results: Majority were female, age range of 17 - 23 years. 2 out of 3 considered ZIKA to be a problem. 25\% thought that ZIKA could strike in the next 6 months. Majority felt that it is the self, family and other organizations responsibility to protect them from ZIKA. However, 1 out of 4 believed "God has the responsibility to protect us". Stigmatization and discrimination of persons affected by ZIKA was endorsed by only 1 out of every 6 respondents. A quarter of the respondents believe that there needs to be safe and legal permission for abortion for the identified women.

Interpretation: The study implies the need and importance of training healthcare professionals on ZIKV Disaster Preparedness.

Keywords: ZIKA; Knowledge; Psychosocial Support Preparedness

\section{Introduction}

ZIKA virus (ZIKV) was originally discovered in a sentinel rhesus macaque in Uganda in 1947 and is endemic in Africa and Asia. The first large outbreak was in 2007, from the Island of Yap (Federated States of Micronesia), followed by French Polynesia in 2013 [1]. ZIKV infection is typically self-limiting and manifests as fever, rash, conjunctivitis, arthralgia [2], and, uncommonly, neurologic syndromes such as the Guillain-Barré syndrome [3].

ZIKV infection in an otherwise healthy person is typically asymptomatic, and for those who do experience them, the symptoms tend to be mild. Symptoms last 2 - 7 days and consist of a combination of fever, skin rash, conjunctivitis, muscle and joint pain, malaise and headache [1]. In 2015, ZIKV gained global atten- tion when Brazil reported a large outbreak and a concerning increase of both microcephaly, a condition where a baby is born with a small head or the head stops growing after birth, and possible association with Guillain-Barré Syndrome (GBS), an acute neuropathy that affects the nerves controlling muscle strength, transmitting pain, temperature, and touch sensations [1].

Mosquito acquisition of the virus likely occurs during a blood meal; after uptake, the virus replicates and is transmitted to a reservoir animal at the next blood meal [4]. Other non-vector modes of ZIKA virus transmission include congenital, perinatal, and sexual [5]. ZIKA virus has been declared a public health emergency. Nearly 1.3 million persons have been affected in Brazil alone and 20 countries or territories have reported local transmission of the virus [5]. 
Modes of travel are faster, due to which there is a strong chance for further spread into Asian countries. Methods of transmission can be through different methods, be it through Aedes mosquitos' bite, conjugal relation between two individuals or during pregnancy from mother to the child. As the chances of the epidemic hitting the country are expected very soon, a robust, multifaceted response is required to reduce the psychosocial burden and to improve the preparedness among the people.

The most vulnerable sections would be women and children during the ZIKA epidemic as they may face fear and distress about their pregnancy in the background of misconceptions. This will have a positive correlation with the emotional and psychosocial wellbeing in these populations. This may lead to other socioeconomic problems for parents and families, reducing their access to care and support and impacting on their livelihoods. Misconceptions about transmission, the cause of the disability, and blaming of the parents may exist, thus compounding feelings of isolation, guilt, stress, and depression that may be felt by the family of the child and child as he/she gets older [1].

The mental health professionals who are involved in the process of psycho-educating the patient and/or family members need to learn to understand and address the issues about ZIKA as a part of their intervention. The ZIKA Toolkit that is produced by the WHO is a cumulative and comprehensive module which brings into light the method of intervening in the situation. But as this epidemic is one of its first kinds in the country the knowledge about the illness is very limited.

Assessing the conditions with respect to the reviewed articles, it is understood that ZIKA is a biological hazard with a severe physical, psychological, social, economic and political burden which later can affect the well-being and quality of life of vulnerable populations at a micro and macro level. The review states that there are no studies emphasizing on educating the Mental Health professionals and/or Health Care Professionals to improve preparedness among individuals who will be the first point of contact on the primary, secondary or tertiary level for mitigation and medical emergency. Hence there is a strong need to explore these areas and assess the level of knowledge.

As per the Down To Earth's report of 2018, India has the largest ZIKA-exposed population in Asia. The country has had 289 Zika cases in 2018, up to from four in 2016 -2017 [6].
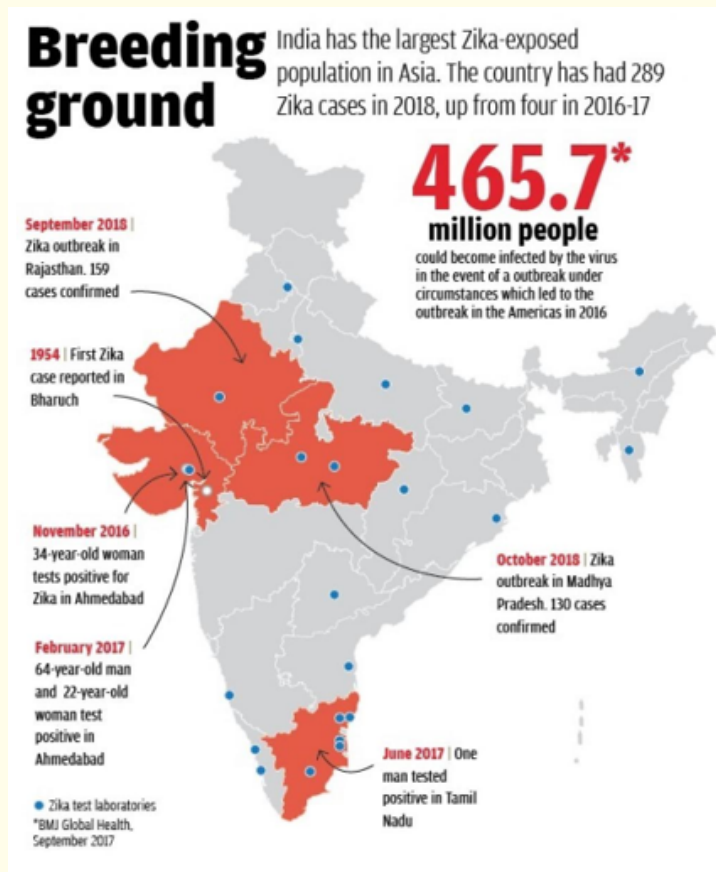

Figure 1

\section{Theorization}

Improving mental health literacy is a significant component of mental health promotion [7]. The concept of health was traditionally seen from a pathogenesis perspective. The pathogenesis approach just looks into the origin of illness and it was found to be not beneficial by the researchers. As opposite to pathogenesis approach, Aaron Antonovsky, a medical sociologist has introduced the theory of salutogenesis [8]. It aims at finding and examining factors which are responsible for the formation and the maintaining of health. The two core concepts of the salutogenesis theory are the Sense of Coherence (SOC) and the Generalized Resistance Resources (GRRs). The sense of coherence is a combination of people's ability to assess and understand the situation they are in, find meaning to move in a health-promoting direction and having the capacity to do so. On the other hand, general resistance is any character in a person, group or environment that can facilitate effective tension management.

\section{Need for the current study}

The review states that there are no studies emphasizing on educating the Mental Health professionals and/or Health Care Professionals to improve preparedness among individuals who will be the 
first point of contact on the primary, secondary or tertiary level for mitigation and medical emergency. Hence there is a strong need to explore these areas and assess the level of knowledge and ways of imparting the same. Hence, it is better to explore the available WHO Toolkit for ZIKA Virus for Health Care Professionals (Section 2) for its adaptability in the Indian settings. The scope of the study extends to the health care workers in primary, secondary and tertiary care settings dealing with women and public health issues. Theoretically, it will bring in a preparedness module in the health and mental health care field to combat a biological disaster.

\section{Materials and Methods}

The study sample considered was the 285 Undergraduate Nursing students at NIMHANS using the Survey method. This population was considered as the Nursing population are the ones who are present at the primary, secondary and tertiary levels and are the first point of contact in any hospital setting.
The sample was assessed across various parameters. An individualized semi-structured interview method was utilized to collect the information. The WHO-KAP semi-structured schedule for ZIKA was used for the study which had 90 questions varying across Knowledge, Attitudes, and Practices of individuals towards ZIKA.

\section{Statistical analysis}

A statistical analysis of the data was done using SPSS 14.0. Descriptive statistics were utilised to describe the data for the study. Wherever relevant, the data has been described further.

\section{Results}

The sample $(\mathrm{n}=285)$ predominantly consisted of females $(89.82 \%)$ in the age range of 18 - 22 years with mean age 19 years in which 78 percent were following Hinduism. 82 percent belonged to Nuclear family and had nearest health facility situated within $2.5 \mathrm{~km}$.

\begin{tabular}{|l|c|c|}
\hline Sl \# & Characteristic Domain & $\mathbf{\%}$ \\
\hline 1 & Risk expected by the individual about the strike of ZIKA in next 6 months & 22 \\
\hline 2 & $\begin{array}{c}\text { Belief that the local healer, traditional birth attendant or a midwife's responsibility } \\
\text { to protect self, household and community from ZIKA }\end{array}$ & 47 \\
\hline 3 & Confused about ZIKA being a problem & 51 \\
\hline 4 & Women should avoid getting pregnant during the time of ZIKA-Not known & 37 \\
\hline 5 & All women who get ZIKA during pregnancy to have access to safe and legal abortion & 49 \\
\hline 6 & Do not know if they would be discriminated or stigmatized because of ZIKA & 38 \\
\hline 7 & Disagree that a woman having a child with Microcephaly or any other disability & 47 \\
\hline 8 & When somebody in the family gets ZIKA should it remain private/ a secret & 38 \\
\hline
\end{tabular}

Table 1: Results.

\section{Discussion}

The current study focused on assessing the level of knowledge among the undergraduate nursing trainees under the College of Nursing, in NIMHANS. Majority of the respondents were female and were contacted through proper channel. The sample consisted of students across the first, second, third and fourth year of Nursing.

During a period of an epidemic, especially in the context of a biological disaster, an individuals' initial instinct is to come to a clinic for a consultation and the first point of contact are the nurses. Gould in the article "Outbreaks of infection in community settings:
The nursing implications" stated that nurses are in a stature where they are able to identify the early signs and symptoms of an infection and help in early treatment intervention. As the symptoms of ZIKA start at mild symptoms with no specific treatment, awareness among the first point of contact becomes important. The same has been concluded by the studies regarding the importance of involving nurses for the awareness and education aspect of ZIKA.

Assessing the conditions with respect to the reviewed articles, it is understood that ZIKA is a biological hazard with a severe physical, psychological, social, economic and political burden which later can affect the well-being and quality of life of vulnerable po- 
pulations at a micro and macro level. Hence, it is understood also from the study that the level of knowledge among the health professionals hereby being the nursing students who work across the primary, secondary and tertiary centers was limited, thereby increasing risk in the time of an epidemic.

At the same time, this being the first study assessing the knowledge of the health professionals in a health care facility gave us the results wherein it was found that 2 out of 3 respondents considered ZIKA to be a problem which correlates with WHO declaring it as a Global Health Emergency [1]. Of the fact that 1 out of 4 thought that ZIKA could strike in another 6 months, it correlates with report that the regions of the country where ZIKA was expected.

Though it is considered that during a national health emergency, it is supposed to be the government's responsibility to protect the individual from the epidemic, majority of the respondents believed that they themselves, their families and other organizations namely the non-funded organization would be the ones who should take up the responsibility of protecting during the period of a ZIKA outbreak. Self-efficacy increases the effectiveness of oral communication and promotes civic participation [9].

However, 1 out of 4 believed "God has the responsibility to protect us", which correlates with the traditional pathways of care that individuals always believe in. A similar biological disaster which had created a furor in the country was that of HIV/AIDS wherein it was found that $50 \%-60 \%$ of paramedical had misconceptions on HIV/AIDS resulting in maladaptive pathways of care [10].Triangulating the information, it becomes more important to help spread proper knowledge through knowledge which shall help in the better attitudes and practices that health care professionals have towards the epidemic.

Stigma and Discrimination as defined by Goffman [11] as the differential treatment provided to individuals during an epidemic or an illness thereby negatively impacting the lives of individuals was endorsed by only 1 out of every 6 respondents, thereby stating that there maybe a reduced stigma level among individuals and thereby not reducing the quality of life of the affected population. This result contradicts the previous results with respect to biological disaster wherein there was a signification relationship established between the attitude of health care providers and social stigmatized attitude [12].
Understanding the fact that the condition has co-morbid conditions of Microcephaly and Guillain-Barré syndrome, a quarter of the respondents believed that women need to have safe and legal permission for choosing abortion in the condition where they are diagnosed with ZIKA Virus infection. The Medical Termination of Pregnancy Act of 1971 with the amendments of 2002 and 2003 does approve medical abortion and is aimed at providing safe services but the problems of the poor awareness of the law, the linked contraceptive targets and the lack of good clinical practice to provide safe access to safe abortion care still remain making it a policy level decision and implementation to ponder upon at the time of the epidemics [13].

\section{Limitations}

The current study focused on understanding the knowledge among the nursing trainees regarding ZIKA, based on their descriptive reports. While providing a descriptive status of the findings, certain methodological limitations may prevail thus. Similarly, the study is based on data collected from the sample only on a single-contact basis. Further contact could have proved helpful in understanding their attitude and practices status better.

\section{Conclusion}

The ZIKA Virus being the first of its kind in the country with the co-morbid conditions has no vaccine or cure as of now. It is tough understood that the health professionals have information regarding how the virus is spread, the prevention strategies and method of management. The socio-cultural aspects increasing the risk behavior is also understood and the effect on the functionality at biological, social and occupational levels can be perceived. The poor understanding and preparedness among the health care population will have higher chances of increasing the Disability-adjusted Life years here and thereby increasing the Global Burden affecting the government and the international organizations. Health Knowledge, awareness and lifestyle modification is the current need of the day. The Nursing profession being the first point of contact, it would be helpful if the section on the current epidemics being of biological origin is included in the curriculum as a section in Microbiology subject. Thereby, making it imperative for the healthcare professionals to increase their knowledge towards the epidemic as a lack of adequate disaster preparedness and facilities for medical and psychiatric care is imminent. 


\section{Bibliography}

1. World Health Organization. Who Toolkit For The Care And Support Of People Affected By Complications Associated With Zika Virus (2017).

2. Brasil P., et al. "Zika virus outbreak in rio de janeiro, brazil: clinical characterization, epidemiological and virological aspects". PLOS Neglected Tropical Diseases 10.4 (2016): e0004636.

3. Parra B., et al. "Guillain-barré syndrome associated with zika virus infection in Colombia". The New England Journal of Medicine 375.16 (2016): 1513-1523.

4. Ioos S., et al. "Current Zika virus epidemiology and recent epidemics". Médecine et Maladies Infectieuses 44.4 .7 (2014): 302-307.

5. Plourde AR and Bloch EM. A literature review of zika virus 22.7 (2016).

6. Down To Earth. How India is responding to its first outbreak (2018).

7. Francis Catherine. Pirkis Jane, Dunt David. "Improving Mental Health Literacy: A Review of the Literature (2002).

8. Gloeckler M. "Where can we discover the basis for the health and well-being of body, soul and spirit?" (2001).

9. Verba S., et al. "Beyond ses: a resource model of political participation". The American Political Science Review 89.2 (1995): 271-94.

10. Dobe M. "Awareness on AIDS among health care professionals". Indian Journal of Public Health 39.3 (1995): 105-108.

11. Goffman E. "Stigma: Notes on the Management of Spoiled Identity". New York: New York: Simon and Schuster Inc (1963).

12. Zarei N., et al. "Stigmatized attitude of healthcare providers: a barrier for delivering health services to hiv positive patients". International Journal of Community Based Nursing and Midwifery 3.4 (2015): 292-300.

13. Hirve SS. "Abortion law, policy and services in India: a critical review". Reproductive Health Matters 12.22 (2004): 114-121.

\section{Assets from publication with us}

- Prompt Acknowledgement after receiving the article

- Thorough Double blinded peer review

- Rapid Publication

- Issue of Publication Certificate

- High visibility of your Published work

Website: https://www.actascientific.com/

Submit Article: https://www.actascientific.com/submission.php

Email us: editor@actascientific.com

Contact us: +919182824667 